\title{
VIRTUAL IMMEDIATE BASINS OF NEWTON MAPS AND ASYMPTOTIC VALUES
}

\author{
XAVIER BUFF AND JOHANNES RÜCKERT
}

\begin{abstract}
Newton's root finding method applied to a (transcendental) entire function $f: \mathbb{C} \rightarrow \mathbb{C}$ is the iteration of a meromorphic function $N_{f}$. It is well known that if for some starting value $z_{0}$, Newton's method converges to a point $\xi \in \mathbb{C}$, then $f$ has a root at $\xi$. We show that in many cases, if an orbit converges to $\xi=\infty$ for Newton's method, then $f$ has a 'virtual root' at $\infty$. More precisely, we show that if $N_{f}$ has an invariant Baker domain that satisfies some mild assumptions, then 0 is an asymptotic value for $f$.

Conversely, we show that if $f$ has an asymptotic value of logarithmic type at 0 , then the singularity over 0 is contained in an invariant Baker domain of $N_{f}$, which we call a virtual immediate basin. We show by way of counterexamples that this is not true for more general types of singularities.
\end{abstract}

\section{INTRODUCTION}

Let $f: \mathbb{C} \rightarrow \mathbb{C}$ be an entire function. Newton's root finding method for $f$ is implemented by iterating the associated Newton map

$$
N_{f}: \mathbb{C} \rightarrow \widehat{\mathbb{C}}, z \mapsto z-\frac{f(z)}{f^{\prime}(z)} .
$$

It is well known that $\xi \in \mathbb{C}$ is a fixed point of $N_{f}$ if and only if $f(\xi)=0$. Furthermore, every finite fixed point $\xi$ of $N_{f}$ is attracting, so it has an invariant neighborhood on which $N_{f}$-orbits converge locally uniformly to $\xi$. In 2003 , Douady raised the following question: if there exists a virtual immediate basin (an invariant, unbounded domain on which $N_{f}$-orbits converge locally uniformly to $\infty$ ), does this imply that $\infty$ is a 'virtual root' of $f$, in other words, does this imply that 0 is an asymptotic value of $f$ ? In this paper, we give a condition under which this is true. A recent result of Bergweiler, Drasin and Langley BDL implies that the condition is sharp when the Julia set of Newton maps is connected. Conversely, we show that if $f$ has a singularity of logarithmic type over 0 , then this singularity is contained in a virtual immediate basin of $N_{f}$; if it is not of logarithmic type, then we provide counterexamples.

The dynamics of $N_{f}$ partitions the Riemann sphere $\widehat{\mathbb{C}}$ into two completely invariant parts: the open Fatou set of all points at which the iterates $\left\{N_{f}^{\circ n}\right\}_{n=0}^{\infty}$ are defined and form a normal family in the sense of Montel, and its complementary Julia set that contains the backward orbit of $\infty$; see

2000 Mathematics Subject Classification. 30D05, 30D99, 37F10, 37F75, 49M15.

Key words and phrases. Newton map, virtual immediate basin, asymptotic value, Baker domain. 
Be1, Mi for an introduction to these concepts. Note that starting values in the Julia set will never converge to an attracting fixed point of $N_{f}$.

A component of the Fatou set of $N_{f}$ for which no point converges to a root of $f$ under iteration is either wandering or will eventually land on a cycle of Böttcher domains, Leau domains, Siegel disks, Herman rings or Baker domains (compare [Be1, Theorem 6]).

The possibilities become much more restricted when considering an invariant component $U$ of the Fatou set, so that $N_{f}(U) \subset U$. In this case, it follows from Proposition 2.1 that $U$ either contains a root of $f$, or is an invariant Herman ring or Baker domain.

Shishikura Sh] has shown that if $N_{f}$ is rational, then its Julia set is connected (see Proposition 2.5 for a characterization of rational Newton maps). It is conjectured that Shishikura's result can be extended to all Newton maps of entire functions. If this is true, an invariant Fatou component of $N_{f}$ either contains a root of $f$ or is a virtual immediate basin (see Section 2 for the precise definition).

In this paper, we continue the analysis of virtual immediate basins in $\mathrm{MS}$ and $\mathrm{RuS}$. We prove that if $f$ has a logarithmic singularity over 0 , then $N_{f}$ has a virtual immediate basin (in 1994, Bergweiler, von Haeseler, Kriete, Meier and Terglane investigated a class of functions $f$ that tend to 0 in a sector and showed that a right end of this sector is contained in a Baker domain of $N_{f}$ BHK, Theorem 3.3]).

For non-logarithmic singularities over 0 , we give examples of functions whose Newton maps do not have a virtual immediate basin associated to these singularities.

Furthermore, we show that there are three classes of virtual immediate basins for $N_{f}$, two of which induce an asymptotic value at 0 for $f$. For the third class, this statement requires an additional assumption, without which it is false. Every such virtual immediate basin even has an open subset of starting values $z_{0}$ such that as $z_{n}=N_{f}^{\circ n}\left(z_{0}\right) \rightarrow \infty, f\left(z_{n}\right) \rightarrow 0$.

Our paper is structured as follows: in Section 2] we give a precise definition of virtual immediate basins and state several of their properties. In Section 3, we recall some fundamental notions concerning singular values. In Section 4, we prove that a logarithmic singularity over 0 for $f$ induces a virtual immediate basin for $N_{f}$, while the counterexamples for direct singularities are treated in Section [5. The converse theorem is stated and proved in Section [6. The underlying idea of the proof is to compare iterates of the Newton map $N_{f}=\mathrm{id}-\frac{f}{f^{\prime}}$ to the time 1 flow of $\dot{z}=-\frac{f(z)}{f^{\prime}(z)}$.

\section{Virtual Immediate Basins}

The concept of a virtual immediate basin was introduced in $\mathrm{MS}$ to explain the behavior of Newton maps between different accesses to $\infty$ of an immediate basin. Examples of Newton maps having virtual immediate basins can be found in $\mathrm{MS}, \mathrm{RuS}$; these example are discussed in detail in Ma. The name was chosen to suggest that these domains behave in many ways similar to immediate basins.

The following proposition characterizes Newton maps of entire functions. 
Proposition 2.1 (Newton Maps). [RuS, Proposition 2.8]. Let $N: \mathbb{C} \rightarrow \widehat{\mathbb{C}}$ be a meromorphic function. It is the Newton map of an entire function $f: \mathbb{C} \rightarrow \mathbb{C}$ if and only if for each fixed point $N(\xi)=\xi \in \mathbb{C}$, there exists a natural number $m>0$ such that $N^{\prime}(\xi)=\frac{m-1}{m}<1$. In this case, there exists $c \neq 0$ such that

$$
f=c \cdot \exp \left(\int \frac{d \zeta}{\zeta-N(\zeta)}\right)
$$

Note that while all definitions in this section are written in terms of Newton maps, they make sense for arbitrary meromorphic functions.

Definition 2.2 (Immediate Basin). Let $N_{f}$ be a Newton map. If $\xi$ is an attracting fixed point of $N_{f}$, we call the open set

$$
\left\{z \in \mathbb{C}: \lim _{n \rightarrow \infty} N_{f}^{\circ n}(z)=\xi\right\}
$$

its basin (of attraction). The component of the basin that contains $\xi$ is called its immediate basin and denoted $U_{\xi}$.

For the definition of virtual immediate basins, we need the following concept.

Definition 2.3 (Absorbing Set). Let $V$ be an $N_{f}$-invariant domain. A connected and simply connected open set $A \subset V$ is called $a$ weakly absorbing set for $V$ if $N_{f}(A) \subset A$ and for each compact $K \subset V$, there exists $k \in \mathbb{N}$ such that $N_{f}^{o k}(K) \subset A$.

We call $A$ an absorbing set if it is weakly absorbing and additionally satisfies $N_{f}(\bar{A}) \subset A$, where the closure is taken in $\mathbb{C}$.

Definition 2.4 (Virtual Immediate Basin). A domain $V \subset \mathbb{C}$ is called a virtual immediate basin for $N_{f}$ if it is maximal (among domains in $\mathbb{C}$ ) with respect to the following conditions:

1. for every $z \in V, \lim _{n \rightarrow \infty} N_{f}^{\circ n}(z)=\infty$;

2. $V$ contains an absorbing set.

Every virtual immediate basin is unbounded, invariant and simply connected [MS, Theorem 3.4]. Since Newton maps of polynomials have a repelling fixed point at $\infty$, virtual immediate basins can appear only for Newton maps of transcendental functions.

Proposition 2.5 (Rational Newton Map). [RuS, Proposition 2.11]. Let $f: \mathbb{C} \rightarrow \mathbb{C}$ be an entire function. Its Newton map $N_{f}$ is rational if and only if there exist polynomials $p, q$ such that $f=p \cdot e^{q}$. In this case, $\infty$ is a repelling or parabolic fixed point.

More precisely, let $m:=\operatorname{deg} p$ and $n:=\operatorname{deg} q$. If $n=0$ and $m \geq 2$, then $\infty$ is repelling with multiplier $\frac{m}{m-1}$. If $n>0$, then $\infty$ is parabolic with multiplier +1 and multiplicity $n+1 \geq 2$.

In the following, let $f$ be a transcendental entire function. If $N_{f}$ is rational, then it has virtual immediate basins which are the attracting petals of the parabolic fixed point at $\infty$ (see [Mi, Theorem 10.5]). If $N_{f}$ is transcendental meromorphic, then any virtual immediate basin is (contained in) an invariant Baker domain. 
Definition 2.6 (Baker Domain). Let $B$ be an invariant component of the Fatou set of $N_{f}$. If $\lim _{n \rightarrow \infty} N_{f}^{\circ n}(z)=\infty \in \partial B$ for all $z \in B$ and $N_{f}$ has an essential singularity at $\infty$, then we call $B$ a Baker domain of $N_{f}$.

If $B$ is a simply connected Baker domain, it contains a weakly absorbing set $A$ by a result of Cowen [Co, Theorem 3.2]. Using Cowen's work, it is easy to find an absorbing subset of $A$, hence $B$ is a virtual immediate basin. Moreover, Cowen's result implies that there are three dynamically defined classes of virtual immediate basins. The following notations are based on Ko] and $[\mathrm{BF}$.

Definition 2.7 (Conformal Conjugacy). Let $V$ be a virtual immediate basin of $N_{f}$ and define $T(z)=z+1$. If there exists a weakly absorbing set $A$ for $V$, a T-invariant domain $\Omega \subset \mathbb{C}$ and a holomorphic map $\phi: V \rightarrow \Omega$ such that

$$
\phi \circ N_{f}(z)=T \circ \phi(z)
$$

for all $z \in V, \phi$ is univalent on $A$ and $\phi(A) \subset \Omega$ is a weakly absorbing set for $\left.T\right|_{\Omega}$, then we call the triple $(\Omega, \phi, T)$ a conformal conjugacy for $N_{f}$ on $V$.

Definition 2.8 (Types of Virtual Immediate Basins). Let $V$ be a virtual immediate basin of $N_{f}$. We say that $V$ is parabolic of type I if it has a conformal conjugacy $(\Omega, \phi, T)$ such that $\Omega=\mathbb{C}$. It is parabolic of type II if there exists a conjugacy such that $\Omega$ is an upper or lower half-plane and hyperbolic with constant $h$ if there exists $h>0$ such that $\Omega$ is the strip

$$
S_{h}:=\{z \in \mathbb{C}:|\operatorname{Im}(z)|<h\} .
$$

Theorem 2.9 (Classification of Virtual Immediate Basins). Co, Theorem 3.2]. Every virtual immediate basin $V$ has a conformal conjugacy and is of exactly one of the three types defined above. If $V$ is hyperbolic, the constant $h$ is uniquely defined.

REMARK. We believe that any Baker domain of a Newton map is simply connected; if this were proved, the notion of a virtual immediate basin would simply stand for either an attracting petal or a Baker domain, depending on whether the map under consideration is rational or not.

\section{Asymptotic Values}

We recall several important definitions concerning the singular values of a meromorphic map. Singular values play an important role in iteration theory, because their orbits determine the dynamics of a map in many ways.

We denote by $B_{r}(z)$ the open disk of radius $r>0$ around $z \in \mathbb{C}$. In this section, let $g: \mathbb{C} \rightarrow \widehat{\mathbb{C}}$ be a meromorphic function.

Definition 3.1 (Regular and Singular Value). Let $a \in \mathbb{C}$ and assume that for $r>0, U_{r}$ is a connected component of $g^{-1}\left(B_{r}(a)\right)$ such that $U_{r_{1}} \subset U_{r_{2}}$ if $r_{1}<r_{2} \cdot{ }^{1}$ We have the following two cases:

\footnotetext{
${ }^{1}$ The function $U: r \mapsto U_{r}$ is completely determined by its germ at 0 . Since $\bigcap_{r>0} U_{r}$ is connected, the intersection contains at most one point.
} 
1. If $\bigcap_{r>0} U_{r}=\{z\}$ for some $z \in \mathbb{C}$, then $g(z)=a$. If $g^{\prime}(z) \neq 0$, then we call $z$ a regular point of $g$. If $g^{\prime}(z)=0$, then $z$ is called a critical point and a a critical value. In this case, we say that the critical point $z$ lies over $a$.

2. If $\bigcap_{r>0} U_{r}=\emptyset$, then we say that $U: r \mapsto U_{r}$ defines a singularity of $f^{-1}$ and we call $a$ an asymptotic value. For simplicity, we call $U$ a singularity and say it lies over $a$.

A singular value is an asymptotic or critical value. If no singularities or critical points lie over a point, we call it a regular value.

Note that there can be many different singularities as well as regular or critical points over any given point $a \in \mathbb{C}$.

For a rational map, all singular values are critical values. Asymptotic values of transcendental maps have a well-known characterization via paths.

Lemma 3.2 (Asymptotic Path). A point $a \in \widehat{\mathbb{C}}$ is an asymptotic value of $g$ if and only if there exists a path $\Gamma:(0, \infty) \rightarrow \mathbb{C}$ with $\lim _{t \rightarrow \infty} \Gamma(t)=\infty$ such that $\lim _{t \rightarrow \infty} g(\Gamma(t))=a$.

We call $\Gamma$ an asymptotic path of $a$. We follow $[\mathrm{BE}]$ in the classification of asymptotic values.

Definition 3.3 (Direct, Indirect and Logarithmic Singularity). Let $U$ be a singularity of $g^{-1}$ lying over $a \in \mathbb{C}$.

If $a \notin g\left(U_{r}\right)$ for some $r>0$, then we call $U$ a direct singularity. Otherwise, $U$ is called an indirect singularity.

A direct singularity $U$ over a is called logarithmic if $g: U_{r} \rightarrow B_{r}(a) \backslash\{a\}$ is a universal covering map for all sufficiently small $r$.

As an example, the positive real axis is an asymptotic path of 0 for the $\operatorname{map} z \mapsto \sin (z) / z$. Since its image assumes this value infinitely many times, it is contained in an indirect singularity over 0 . For $z \mapsto \exp z$, any left half plane is a logarithmic singularity over 0 .

\section{A Criterion for Virtual Immediate Basins}

Our first result is the following.

Theorem 4.1 (Logarithmic Singularity Implies Virtual Immediate Basin). Let $f: \mathbb{C} \rightarrow \mathbb{C}$ be an entire function with a logarithmic singularity $U$ over 0 . Then there exists $r_{0}>0$ such that $U_{r_{0}}$ is an absorbing set for a parabolic virtual immediate basin of type I for $N_{f}$.

Note that if $U$ is an indirect singularity, each $U_{r}$ contains infinitely many roots of $f$ and hence infinitely many attracting fixed points of $N_{f}$. Therefore, $U_{r}$ cannot be part of a virtual immediate basin. In Section [5] we show that there exist functions $f: \mathbb{C} \rightarrow \mathbb{C}$ with a direct singularity $U$ over 0 which does not induce a virtual immediate basin for $N_{f}$.

Proof. The idea is to compare the iterates of $N_{f}$ to the time 1 flow of the differential equation $\dot{z}=-\frac{f(z)}{f^{\prime}(z)}$. If $r$ is small enough, this flow sends $U_{r}$ isomorphically to $U_{r / e}$. We will see that for $r$ small enough, $N_{f}$ maps $U_{r}$ 
univalently into itself and is an absorbing set for a virtual immediate basin of $N_{f}$.

First, let $r>0$ be small enough so that $f: U_{r} \rightarrow B_{r}(0) \backslash\{0\}$ is a universal covering. Set $\eta:=-\log r$ and $\mathbb{H}_{\eta}:=\{w \in \mathbb{C}: \operatorname{Re}(w)>\eta\}$. Since $e^{- \text {id }}$ : $\mathbb{H}_{\eta} \rightarrow B_{r}(0) \backslash\{0\}$ is also a universal covering, the map $-\log (f): U_{r} \rightarrow \mathbb{H}_{\eta}$ is biholomorphic with inverse $\psi: \mathbb{H}_{\eta} \rightarrow U_{r}$ (see Figure 1). With this, we get $\log (f(\psi(w)))=-w$ for $w \in \mathbb{H}_{\eta}$.

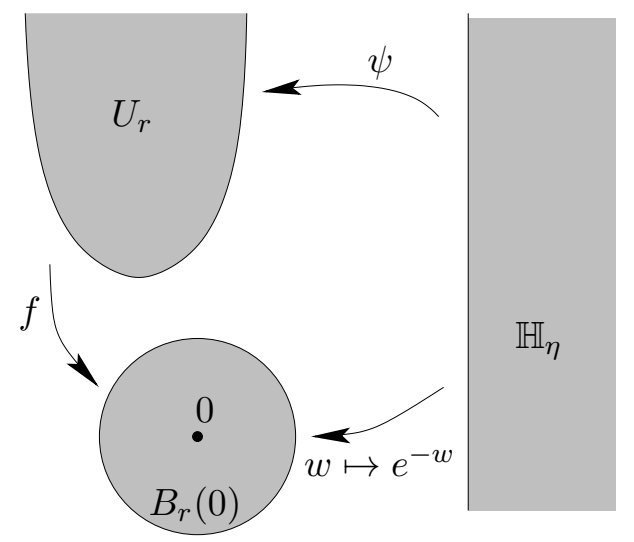

FiguRE 1. If $f: U_{r} \rightarrow B_{r}(0) \backslash\{0\}$ is a universal covering, there exists a biholomorphic map $\psi: \mathbb{H}_{\eta} \rightarrow U_{r}$.

Taking derivatives yields

$$
\frac{f^{\prime}(\psi(w))}{f(\psi(w))} \cdot \psi^{\prime}(w)=-1 ; \quad \text { hence } \quad \psi^{\prime}(w)=-\frac{f(\psi(w))}{f^{\prime}(\psi(w))} .
$$

In other words, $\psi$ is a solution of $\dot{z}=-\frac{f(z)}{f^{\prime}(z)}$ and following the flow during time 1 maps $U_{r}=\psi\left(\mathbb{H}_{\eta}\right)$ to $U_{r / e}=\psi\left(\mathbb{H}_{\eta+1}\right)$.

We now want to compare $N_{f}$ to the time 1 flow of $\dot{z}=-\frac{f(z)}{f^{\prime}(z)}$. We will do the comparison in time space: we will show that if $z=\psi(w)$ with $\operatorname{Re}(w)$ large enough, then $N_{f}(z)=\psi\left(w^{\prime}\right)$ with $w^{\prime}$ close to $w+1$. More precisely, we have the following lemma.

Lemma 4.2. There exists $\eta_{0}>\eta$ and a holomorphic map $G: \mathbb{H}_{\eta_{0}} \rightarrow$ $\mathbb{H}_{\eta_{0}+1 / 2}$ such that for all $w \in \mathbb{H}_{\eta_{0}}$, we have

$$
N_{f} \circ \psi(w)=\psi \circ G(w) \quad \text { and } \quad|G(w)-(w+1)|<\frac{1}{2} .
$$

The proof of Theorem 4.1 is then easily completed. Indeed, set $V_{0}:=$ $\psi\left(\mathbb{H}_{\eta_{0}}\right)=U_{r_{0}}$ with $r_{0}=e^{-\eta_{0}}$ and let $V_{n+1}$ be the component of $N_{f}^{-1}\left(V_{n}\right)$ that contains $V_{0}$. Since all points in $\mathbb{H}_{\eta_{0}}$ converge to $\infty$ under iteration of $G$ (the real part increases by at least $1 / 2$ in each step), we conclude that $V:=\bigcup_{n \in \mathbb{N}} V_{n}$ is a virtual immediate basin of $N_{f}$ with absorbing set $V_{0}$.

Let us now prove Lemma 4.2. Note that

$$
N_{f}(\psi(w))=\psi(w)-\frac{f(\psi(w))}{f^{\prime}(\psi(w))}=\psi(w)+\psi^{\prime}(w) .
$$


Thus, it is equivalent to prove that there exists $\eta_{0}>\eta$ and a holomorphic map $G: \mathbb{H}_{\eta_{0}} \rightarrow \mathbb{H}_{\eta_{0}+1 / 2}$ such that for all $w \in \mathbb{H}_{\eta_{0}}$, we have

$$
\psi(w)+\psi^{\prime}(w)=\psi(G(w)) \text { and }|G(w)-(w+1)|<\frac{1}{2} .
$$

Given $w \in \mathbb{H}_{\eta+2}$, define functions $g, h: B_{2}(w) \rightarrow \mathbb{C}$ by

$$
g: \zeta \mapsto \frac{\psi(\zeta)-\psi(w)-\psi^{\prime}(w)}{\psi^{\prime}(w)} \text { and } h: \zeta \mapsto \zeta-(w+1) .
$$

Since $g$ and $h$ satisfy $g(w)=h(w)=-1, g^{\prime}(w)=h^{\prime}(w)=1$ and can both be extended to all of $\mathbb{H}_{\eta}$ as univalent maps, by Koebe's distortion theorem there exists $\eta_{0}>\eta+2$ such that for every $w \in \mathbb{H}_{\eta_{0}}$ and every $\zeta \in B_{2}(w)$, $|g(\zeta)-h(\zeta)|<1 / 4$.

Clearly, $h(w+1)=0$. Note that $|h(\zeta)|=1 / 2>|g(\zeta)-h(\zeta)|$ when $\zeta$ belongs to the circle $\partial B_{1 / 2}(w+1)$. By Rouché's theorem, the map $g$ has a (unique) root $\xi_{w} \in B_{1 / 2}(w+1)$. It is now easy to see that the map $G: \mathbb{H}_{\eta_{0}} \rightarrow \mathbb{C}$ defined by $G(w)=\xi_{w}$ satisfies equations (10).

\section{A Direct Singularity Counterexample}

In this section, we will exhibit examples of entire functions with direct singularities over 0 that do not induce Baker domains of the associated Newton maps. This shows that Theorem 4.1 cannot be improved much further; 0 is an omitted value in all examples, so that a generalization is not even possible to this case. We will only treat the first example in full detail.

For $\alpha \in] 0,+\infty\left[\right.$, consider the entire function $f_{\alpha}$ defined by

$$
f_{\alpha}(Z)=\exp \left(-\frac{1}{\alpha}\left(Z+\frac{1}{2 i \pi} e^{2 i \pi Z}\right)\right) \text {. }
$$

The function $f_{\alpha}$ has infinitely many singularities over 0 which are necessarily direct since $f_{\alpha}$ does not vanish. We have two kinds of asymptotic paths:

1. for $k \in \mathbb{Z}$, as $t \in \mathbb{R} \rightarrow+\infty, f_{\alpha}\left(k+\frac{1}{4}-i t\right) \rightarrow 0$;

2. as $t \in \mathbb{R} \rightarrow+\infty, f_{\alpha}(t) \rightarrow 0$.

The singularities of the first kind are of logarithmic type. Thus, each one induces a Baker domain of parabolic type I for the Newton map

$$
N_{\alpha}(Z)=Z+\frac{\alpha}{1+e^{2 i \pi Z}} .
$$

The singularity of the second kind is not of logarithmic type and contains infinitely many critical points of $f$. We will see that for some values of $\alpha$, it does not induce a Baker domain for $N_{\alpha}$.

More precisely, observe that $N_{\alpha}(Z+1)=N_{\alpha}(Z)+1$. It follows that we can study the dynamics of $N_{\alpha}$ modulo 1 . In other words, we have

$$
e^{2 i \pi N_{\alpha}(Z)}=g_{\alpha}\left(e^{2 i \pi Z}\right) \quad \text { with } \quad g_{\alpha}(z)=z e^{2 i \pi \alpha /(1+z)} .
$$

The map $g_{\alpha}$ has a fixed point with multiplier $e^{2 i \pi \alpha}$ at $z=0$, a fixed point with multiplier 1 at $z=\infty$ and an essential singularity at $z=-1$.

Let $\mathcal{F}\left(N_{\alpha}\right)$ and $\mathcal{F}\left(g_{\alpha}\right)$ be the Fatou sets of $N_{\alpha}$ and $g_{\alpha}$ and let $\pi: \mathbb{C} \rightarrow \mathbb{C}^{*}$ be the universal covering $\pi: Z \mapsto z=e^{2 i \pi Z}$. We claim that

$$
\mathcal{F}\left(N_{\alpha}\right)=\pi^{-1}\left(\mathcal{F}\left(g_{\alpha}\right)\right) \text {. }
$$


It is easy to see that $\pi^{-1}\left(\mathcal{F}\left(g_{\alpha}\right)\right) \subset \mathcal{F}\left(N_{\alpha}\right)$ (see for example Be2]). The inclusion $\mathcal{F}\left(N_{\alpha}\right) \subset \pi^{-1}\left(\mathcal{F}\left(g_{\alpha}\right)\right)$ is less immediate. One may argue as follows. Assume $z_{0}=\pi\left(Z_{0}\right) \notin \mathcal{F}\left(g_{\alpha}\right)$. Then, $z_{0}$ lies in the closure of the set of iterated $g_{\alpha}$-preimages of -1 (otherwise, the family of iterates of $g_{\alpha}$ would be well defined near $z_{0}$ and avoid the infinite set $g_{\alpha}^{-1}(\{-1\})$, thus it would be normal). It follows that any neighborhood of $Z_{0}$ contains a preimage of a pole of $N_{\alpha}$. Thus, $Z_{0} \notin \mathcal{F}\left(N_{\alpha}\right)$.

As $z \rightarrow \infty$, we have

$$
g_{\alpha}(z)=z+1+\frac{2 i \pi \alpha}{z}+o(1 / z) .
$$

Thus, the parabolic fixed point at $\infty$ has multiplicity 2 . It has a single attracting direction along the positive real axis. The full preimage of its parabolic basin under the map $e^{2 i \pi Z}$ is the union of the Baker domains of $N_{\alpha}$ induced by the singularities of $f_{\alpha}$ of the first kind. The map $g_{\alpha}$ has exactly two critical points: the solutions to $(1+z)^{2}-2 i \pi \alpha z=0$.

Conjugating with $z \mapsto w=1 /(z+1)$, we may put the singularity at $\infty$ and the fixed points at 0 and 1 . The map $g_{\alpha}$ is thus conjugate to the meromorphic function

$$
h_{\alpha}(w)=\frac{w}{w+(1-w) e^{2 i \pi \alpha w}} .
$$

The map $h_{\alpha}$ has growth order 1 and two critical points. Thus, it has at most 2 asymptotic values by [BE, Corollary 3]. But as $t \in \mathbb{R} \rightarrow+\infty$, $h_{\alpha}(i t) \rightarrow 0$ and $h_{\alpha}(-i t) \rightarrow 1$. Thus, $h_{\alpha}$ has exactly 2 (fixed) asymptotic values and 2 critical values and is therefore a finite type map. It is well known that finite type meromorphic functions have neither wandering domains nor Baker domains BKL, RS].

The map $h_{\alpha}$ has a fully invariant parabolic point at 0 and for suitably chosen $\alpha$, the fixed point at 1 is Cremer (in analogy to [Mi, Theorem 11.13]). We want to prove that in this case, the Fatou set of $h_{\alpha}$ consists of the parabolic basin at 0 and its preimage components. We deduce that then, the Fatou set of $g_{\alpha}$ is equal to the parabolic basin of $\infty$ and its preimage components. Thus, every Fatou component of $N_{\alpha}$ maps after finitely many iterations into one of the invariant Baker domains induced by the first kind of singularities of $f_{\alpha}$. There is no Fatou component associated to the second kind of singularity of $f_{\alpha}$.

So it remains to show that $h_{\alpha}$ has no additional non-repelling periodic points nor Herman rings. While both claims follow directly from Epstein's version of the Fatou-Shishikura inequality for finite type maps [E1, E2, E3], we provide a version of Epstein's proof that is sufficient for our purposes; we treat Herman rings separately in Lemma 5.2

Lemma 5.1 (Epstein). There cannot be any additional non-repelling periodic points.

Proof. Suppose that $h_{\alpha}$ has an additional non-repelling cycle

$$
\left\{z_{1} \mapsto z_{2} \mapsto \ldots, \mapsto z_{k} \mapsto z_{1}\right\}
$$

Let $v_{1}$ and $v_{2}$ be the two critical values of $h_{\alpha}$, set

$$
X=\left\{0,1, z_{1}, \ldots, z_{k}\right\}, \quad X^{\prime}=X \cup\left\{v_{1}, v_{2}\right\} .
$$


Let $\mathcal{Q}^{1}(X)$ (resp. $\mathcal{Q}^{1}\left(X^{\prime}\right)$ ) be the set of meromorphic quadratic differentials on $\widehat{\mathbb{C}}$ which are holomorphic outside $X$ (resp. $X^{\prime}$ ) and have at most simple poles in $X$ (resp. $\left.X^{\prime}\right)$. Let $\mathcal{Q}^{2}(X)$ be the set of meromorphic quadratic differentials on $\widehat{\mathbb{C}}$ which are holomorphic outside $X$, have at most double poles in $X$ and whose polar part of order 2 along $X$ is of the form

$$
A \frac{d z^{2}}{z^{2}}+B \frac{d z^{2}}{(z-1)^{2}}+C \sum_{i=1}^{k} \frac{d z^{2}}{\left(z-z_{i}\right)^{2}} \quad \text { with } A, B, C \in \mathbb{C} .
$$

The sets $\mathcal{Q}^{1}(X), \mathcal{Q}^{1}\left(X^{\prime}\right)$ and $\mathcal{Q}^{2}(X)$ are vector spaces of respective dimensions $k-3, k-1$ and $k$. We can define a linear map $\nabla: \mathcal{Q}^{2}(X) \rightarrow \mathcal{Q}^{1}\left(X^{\prime}\right)$ as follows. If $U$ is a simply connected subset of $\widehat{\mathbb{C}} \backslash X^{\prime}$, then $h_{\alpha}: h_{\alpha}^{-1}(U) \rightarrow U$ is a (trivial) covering map. We let $\left(g_{i}: U \rightarrow \widehat{\mathbb{C}}\right)_{i \in I}$ be the countably many inverse branches and we set

$$
\left.\left(h_{\alpha}\right)_{*} q\right|_{U}=\left(\sum_{i \in I} g_{i}^{*} q\right) .
$$

The sum is convergent because

$$
\sum_{i \in I} \int_{U}\left|g_{i}^{*} q\right|=\int_{h_{\alpha}^{-1}(U)}|q|<\infty
$$

We can define in such a way a quadratic differential $\left(h_{\alpha}\right)_{*} q$ which is holomorphic outside $X^{\prime}$. A local analysis shows that

$$
\nabla q:=\left(h_{\alpha}\right)_{*} q-q
$$

has at most simple poles at points of $X^{\prime}$ and thus, belongs to $\mathcal{Q}^{1}\left(X^{\prime}\right)$.

Since the dimension of $\mathcal{Q}^{1}\left(X^{\prime}\right)$ is less than the dimension of $\mathcal{Q}^{2}(X)$, the linear map $\nabla$ is not injective and there is a $q \in \mathcal{Q}^{2}(X)$ such that $\nabla q=0$, i.e., $\left(h_{\alpha}\right)_{*} q=q$. To see that this is not possible, set

$$
U_{\varepsilon}:=D(0, \varepsilon) \cup D(1, \varepsilon) \cup \bigcup_{i=1}^{k} h_{\alpha}^{-i}\left(D\left(z_{1}, \varepsilon\right)\right), \quad V_{\varepsilon}:=h_{\alpha}^{-1}\left(U_{\varepsilon}\right),
$$

let $W_{\varepsilon} \subset \widehat{\mathbb{C}} \backslash\left(U_{\varepsilon} \cup\left\{v_{1}, v_{2}\right\}\right)$ be a simply connected subset of full measure and let $g_{i}: W_{\varepsilon} \rightarrow \widehat{\mathbb{C}}$ be the countably many inverse branches of $h_{\alpha}$. Then, for $\varepsilon$ sufficiently small, we have

$$
\int_{\widehat{\mathbb{C}} \backslash U_{\varepsilon}}\left|\left(h_{\alpha}\right)_{*} q\right|=\int_{W_{\varepsilon}}\left|\sum_{i} g_{i}^{*} q\right| \leq \sum_{i} \int_{W_{\varepsilon}}\left|g_{i}^{*} q\right|=\int_{\mathbb{C} \backslash V_{\varepsilon}}|q|
$$

with equality if and only if each $g_{i}^{*} q$ is a (real positive) multiple of $\left(h_{\alpha}\right)_{*} q=q$. In particular $q=h_{\alpha}^{*}\left(g_{i}^{*} q\right)$ has to be locally, and thus globally, a constant multiple of $h_{\alpha}^{*} q$, i.e. $q=c \cdot h_{\alpha}^{*} q$ for some constant $c>0$. But in that case $g_{i}^{*} q=c \cdot q$ and the sum $\sum_{i} \int_{W_{\varepsilon}}\left|g_{i}^{*} q\right|$ will be diverging which is not the case. Thus,

$$
\int_{\widehat{\mathbb{C} \backslash U_{\varepsilon}}}|q| \leq \int_{\widehat{\mathbb{C}} \backslash V_{\varepsilon}}|q|-C_{\varepsilon} \quad \text { with } C_{\varepsilon}>0
$$


Note that for $\delta<\varepsilon$, we have

$$
\begin{aligned}
\int_{\widehat{\mathbb{C}} \backslash U_{\delta}}|q| & =\int_{\widehat{\mathbb{C} \backslash U_{\varepsilon}}}|q|+\int_{U_{\varepsilon} \backslash U_{\delta}}|q| \\
& \leq \int_{\widehat{\mathbb{C} \backslash V_{\varepsilon}}}|q|-C_{\varepsilon}+\int_{V_{\varepsilon} \backslash V_{\delta}}|q| \\
& =\int_{\widehat{\mathbb{C}} \backslash V_{\delta}}|q|-C_{\varepsilon},
\end{aligned}
$$

thus

$$
\int_{\widehat{\mathbb{C}} \backslash V_{\delta}}|q|-\int_{\widehat{\mathbb{C}} \backslash U_{\delta}}|q| \geq C_{\varepsilon}>0 .
$$

We will obtain a contradiction by proving

$$
\liminf _{\delta \rightarrow 0}\left(\int_{\widehat{\mathbb{C} \backslash V_{\delta}}}|q|-\int_{\widehat{\mathbb{C}} \backslash U_{\delta}}|q|\right) \leq 0 .
$$

This is the place where we use the fact that the cycle is non-repelling. As $\delta \rightarrow 0$, we can find a radius $r_{\delta}=\delta+o(\delta)$ such that

$$
D\left(0, r_{\delta}\right) \cup D\left(1, r_{\delta}\right) \cup D\left(z_{1}, r_{\delta}\right) \cup \bigcup_{i=2}^{k} h_{\alpha}^{-i}\left(D\left(z_{1}, \delta\right)\right) \subset V_{\delta} .
$$

Then, $U_{\delta} \backslash V_{\delta}$ is contained within the union of three annuli

$$
\left\{z ; r_{\delta} \leq|z|<\delta\right\} \cup\left\{z ; r_{\delta} \leq|z-1|<\delta\right\} \cup\left\{z ; r_{\delta} \leq\left|z-z_{1}\right|<\delta\right\} .
$$

Since $q$ has at most double poles at 0,1 and $z_{1}$, the integral of $|q|$ on those annuli tends to 0 as $\delta$ tends to 0 and we have

$$
\int_{\widehat{\mathbb{C} \backslash V_{\delta}}}|q|-\int_{\widehat{\mathbb{C} \backslash U_{\delta}}}|q|=\int_{U_{\delta} \backslash V_{\delta}}|q|-\int_{V_{\delta} \backslash U_{\delta}}|q| \leq \int_{U_{\delta} \backslash V_{\delta}}|q| \underset{\delta \rightarrow 0}{\longrightarrow} 0 .
$$

Lemma 5.2. There cannot be any cycle of Herman rings.

Proof. Recall that 0 is a multiple fixed point and its immediate basin of attraction must contain a critical point $\omega_{0}$ and the critical value $v_{0}=h_{\alpha}\left(\omega_{0}\right)$. Also, 1 is a Cremer point. It must be accumulated by the orbit of the second critical point $\omega_{1}$ with critical value $v_{1}=h_{\alpha}\left(\omega_{1}\right)$.

Assume there is a cycle of Herman rings $H_{1} \mapsto H_{2} \mapsto \ldots \mapsto H_{k} \mapsto H_{1}$. Let $\Gamma$ be the union of the equators of the Herman rings $H_{i}$ ( $\Gamma$ is the union of a cycle of Jordan curves). Choose a connected component $W$ of $\widehat{\mathbb{C}} \backslash \Gamma$ which does not contain 1 . Then, there are infinitely many iterates of $v_{1}$ contained in $W$ (accumulating a boundary component of some Herman ring). In particular, there is an integer $m>2$ such that $h_{\alpha}^{\circ m}\left(v_{1}\right) \in W$. Let $D$ be a disk around 1 avoiding $\Gamma$, the forward orbit of $v_{0}$ and the $m$ first iterates of $v_{1}$. Let $D_{-1}$ be the connected component of $h_{\alpha}^{-1}(D)$ containing 1. Since $D \backslash\{1\}$ does not contain any singular value of $h_{\alpha}, h_{\alpha}: D_{-1} \rightarrow D$ has to be an isomorphism. Since $D_{-1}$ contains 1 and avoids $\Gamma$, it does not contain $h_{\alpha}^{\circ m}\left(v_{1}\right)$. So, $D_{-1}$ is a disk avoiding $\Gamma$, the forward orbit of $v_{0}$ and the $m$ first iterates of $v_{1}$. We can therefore construct inductively a sequence of disks $D_{-k}$ containing 1 such that $h_{\alpha}^{\circ k}: D_{-k} \rightarrow D$ is an isomorphism. Since 
$\left|\left(h_{\alpha}^{\circ k}\right)^{\prime}(1)\right|=1$ for all $k \in \mathbb{N}$, by Koebe's one quarter theorem the disks $D_{-k}$ contain a common neighborhood of 1 on which the iterates of $h_{\alpha}$ form a normal family. This contradicts the fact that 1 is a Cremer point contained in the Julia set of $h_{\alpha}$.

Note that if we choose $\alpha \in \mathbb{Q}, N_{\alpha}$ will have a wandering domain that projects to a parabolic basin of a parabolic fixed point. If $\alpha$ is a Brjuno number, $N_{\alpha}$ will have a univalent Baker domain of parabolic type II which projects to a Siegel disk of $g_{\alpha}$.

We can construct other examples in a similar way. The maps we will present do not have fixed points. It follows from Proposition 2.1 that they are Newton maps of non-vanishing entire functions, whose singularities over 0 are therefore direct.

Assume

$$
N(Z)=Z+\frac{\alpha}{1+\varepsilon \sin (2 \pi Z)}
$$

with

$$
0<\varepsilon<1 \text { and } 0<\alpha<m_{\varepsilon}=\left\lfloor\frac{(1-\varepsilon)^{2}}{2 \pi \varepsilon}\right\rfloor .
$$

Then, $N$ is the Newton map of an entire function $f$ such that $f(t) \rightarrow 0$ as $t \in \mathbb{R} \rightarrow+\infty$. The restriction of $N$ to $\mathbb{R}$ is an increasing homeomorphism which commutes with translation by 1 . Indeed,

$$
N^{\prime}(Z)=1-\frac{2 \pi \varepsilon \alpha \cos (2 \pi Z)}{(1+\varepsilon \sin (2 \pi Z))^{2}} \geq 1-\frac{2 \pi \varepsilon \alpha}{(1-\varepsilon)^{2}}>0 .
$$

Thus, it has a well defined rotation number $\operatorname{Rot}(N)$. This rotation number is positive since $N(Z)>Z$. Note that for $\alpha=m_{\varepsilon}, N(0)=m_{\varepsilon}$ and thus, $\operatorname{Rot}(N)=m_{\varepsilon}$. For each fixed $\varepsilon \in(0,1)$, the rotation number increases continuously from 0 to $m_{\varepsilon}$ as $\alpha$ increases from 0 to $m_{\varepsilon}$. If $\operatorname{Rot}(N)$ is rational, then $N$ has a chain of wandering domains along the real axis. If $\operatorname{Rot}(N)$ is a Brjuno number, $N$ has a univalent Baker domain of hyperbolic type centered on the real axis. For suitably chosen parameters $\alpha, \operatorname{Rot}(N)$ is irrational and the induced map $N: \mathbb{R} / \mathbb{Z} \rightarrow \mathbb{R} / \mathbb{Z}$ is topologically but not analytically conjugate to the rotation $Z \mapsto Z+\operatorname{Rot}(N): \mathbb{R} / \mathbb{Z} \rightarrow \mathbb{R} / \mathbb{Z}$. It should follow that $N$ does not have any Baker domain associated to the singularity of $f$ containing the large positive real numbers. The proof should be similar to the one we presented above: study the dynamics modulo 1 .

In the previous examples, $f$ had a direct singularity containing critical points of $f$. One may wonder whether it is the presence of critical points that prevents $N_{f}$ from having a Baker domain associated to the singularity. The following example shows that this is not the case. We still assume $\alpha>0$ and set

$$
N_{\alpha}(Z)=Z+\alpha e^{e^{2 i \pi Z}} .
$$

Then, $N_{\alpha}$ does not have any fixed points. So, it is the Newton map of the non-vanishing entire function

$$
f_{\alpha}(Z)=\exp \left(-\frac{1}{\alpha} \int_{0}^{Z} e^{-e^{2 i \pi W}} d W\right) .
$$


Note that when $W \in \mathbb{R}$, the real part of $e^{-e^{2 i \pi W}}$ is greater than $1 / e$. Thus, for $\alpha>0$ and for $t \in[0,+\infty)$, we have

$$
\left|f_{\alpha}(t)\right| \leq e^{-t /(e \alpha) \underset{t \rightarrow+\infty}{\longrightarrow} 0 .}
$$

The entire map $f_{\alpha}$ has a singularity over 0 containing large real numbers. This is a direct singularity since $f_{\alpha}$ does not vanish. In addition, $N_{\alpha}$ does not have poles and so, $f_{\alpha}$ does not have critical points.

Again, $N_{\alpha}(Z+1)=N_{\alpha}(Z)+1$ and $N_{\alpha}$ projects via $Z \mapsto z=e^{2 i \pi Z}$ to an entire map $g_{\alpha}$ fixing 0 with multiplier $e^{2 i \pi \alpha}$ :

$$
g_{\alpha}(z)=z e^{2 i \pi \alpha e^{z}} \text {. }
$$

By a result of Bergweiler $\mathrm{Be} 2$, the Fatou sets of $N_{\alpha}$ and $g_{\alpha}$ correspond under the map $Z \mapsto e^{2 i \pi Z}$. If $g_{\alpha}$ has a Siegel disk around 0, the map $N_{\alpha}$ has a Baker domain of parabolic type II which corresponds to the singularity of $f_{\alpha}$ described above. But if $g_{\alpha}$ has a Cremer point at 0 , there can be no Baker domain for $N_{\alpha}$ associated to the singularity of $f_{\alpha}$ described above.

\section{A Virtual Immediate Basin Implies an Asymptotic Value}

Theorem 6.1 (Virtual Immediate Basin Contains Asymptotic Path). Let $f: \mathbb{C} \rightarrow \mathbb{C}$ be an entire function such that its Newton map $N_{f}$ has a virtual immediate basin $V$. If $V$ is parabolic of type $I$ or type II, then 0 is an asymptotic value of $f$ with asymptotic path in $V$. There exists $H>0$ such that the same is true if $V$ is hyperbolic with constant $h \geq H$.

Bergweiler, Drasin and Langley have constructed an entire function for which 0 is not an asymptotic value and whose Newton map has a virtual immediate basin of hyperbolic type [BDL. Thus, the statement of Theorem 6.1 cannot be extended to all hyperbolic virtual immediate basins.

Using Theorem 6.1 we can give the following formulation of Theorem 5.1 in $[\mathrm{RuS}$.

Corollary 6.2 (Outside Immediate Basins). Let $N_{f}$ be the Newton map of an entire function $f$ and $U_{\xi}$ the immediate basin of the attracting fixed point $\xi \in \mathbb{C}$ for $N_{f}$. Let $\Gamma_{1}, \Gamma_{2} \subset U_{\xi}$ be two $N_{f}$-invariant curves connecting $\xi$ to $\infty$ such that $\Gamma_{1}$ and $\Gamma_{2}$ are non-homotopic in $U_{\xi}$ and let $\widetilde{V}$ be an unbounded component of $\mathbb{C} \backslash\left(\Gamma_{1} \cup \Gamma_{2}\right)$. If the set $N_{f}^{-1}(\{z\}) \cap \widetilde{V}$ is finite for all $z \in \widehat{\mathbb{C}}$, then $\left.f\right|_{\widetilde{V}}$ assumes the value 0 or has 0 as an asymptotic value.

Proof. If $0 \notin f(\widetilde{V})$, then the virtual immediate basin constructed in the proof of [RuS, Theorem 5.1] is parabolic of type I.

For the proof of Theorem 6.1, we will need the following corollary to the Koebe distortion theorem. We thank Dierk Schleicher for pointing it out to us.

Lemma 6.3 (Bounded Non-Linearity). Let $R>0, g: B_{R}(0) \rightarrow \mathbb{C}$ be univalent and $\varepsilon>0$. If $r / R$ is sufficiently small, then

$$
\left|\frac{g(z)-g(w)}{g^{\prime}(z)(z-w)}-1\right|<\varepsilon
$$

for all $w, z \in B_{r}(0)$. 
Proof. By possibly conjugating $g$ with $z \mapsto R z$, multiplying $g$ with a constant or adding a constant to $g$, we may assume that $R=1, g(0)=0$ and $g^{\prime}(0)=1$. Fix $0<r<1$. By the Koebe distortion theorem, there is an $\alpha>0$ independent of $g$ such that

$$
\left|g(z)-g(w)-(z-w) g^{\prime}(z)\right|<\alpha|(z-w)|^{2}
$$

for all $z, w \in B_{r}(0)$ (Taylor expansion around $z$ ). Moreover, there is a $\beta>0$ so that $\left|g^{\prime}(z)\right|>\beta$ for all $z \in B_{r}(0)$. This yields

$$
\left|\frac{g(z)-g(w)}{g^{\prime}(z)(z-w)}-1\right|<\alpha\left|\frac{z-w}{g^{\prime}(z)}\right|<\frac{2 \alpha r}{\beta} .
$$

It follows from the Koebe distortion theorem that $\alpha \rightarrow 0$ and $\beta \rightarrow 1$ as $r \rightarrow 0$. The claim follows.

Proof of Theorem [6.1. Suppose first that $V$ is parabolic of type I. Then, there exists a weakly absorbing set $A$ of $V$ and a conformal conjugacy $(\mathbb{C}, \phi, T)$ such that $F:=\phi(A)$ is an absorbing set for $T: z \mapsto z+1$ in $\mathbb{C}$. Since $\left.\phi\right|_{A}$ is univalent, it has a univalent inverse $\psi: F \rightarrow A$. With this, we get for $z \in F$ that $N_{f}(\psi(z))=\psi(z+1)$, and hence

$$
\psi(z)-\frac{f(\psi(z))}{f^{\prime}(\psi(z))}=\psi(z+1) .
$$

It follows that

$$
\frac{f^{\prime}(\psi(z))}{f(\psi(z))} \cdot(\psi(z+1)-\psi(z))=-1
$$

(note that since $V$ is a virtual immediate basin, $f$ has no roots in $\psi(F)$ ). Let $0<\varepsilon<1$. By Lemma 6.3, there exists $R>2$ such that if $B_{R}(z) \subset F$, then

$$
\left|\frac{\psi^{\prime}(z)}{\psi(z+1)-\psi(z)}-1\right|<\varepsilon,
$$

and by equation (2) and inequality (3) we get

$$
\left|\frac{f^{\prime}(\psi(z))}{f(\psi(z))} \cdot \psi^{\prime}(z)+1\right|=\left|\frac{f^{\prime}(\psi(z))}{f(\psi(z))} \cdot \psi^{\prime}(z) \cdot \frac{\psi(z+1)-\psi(z)}{\psi(z+1)-\psi(z)}+1\right|<\varepsilon .
$$

Since $F$ contains all sufficiently far right translates of the disk $B_{R}\left(z_{0}\right)$, for every $z_{0} \in F$ there exists $S_{z_{0}} \geq 0$ such that (4) holds for all $z_{0}+t$ with real $t \geq S_{z_{0}}$.

Let $z_{0} \in F$ such that $S_{z_{0}}=0$. Then, for $t \geq 0$ and $z=z_{0}+t \in F$, we use a standard estimate in complex variables and inequality (4) to get

$$
\begin{aligned}
|\log (f(\psi(z)))+z| & \leq\left|\int_{z_{0}}^{z}\left((\log \circ f \circ \psi)^{\prime}(\zeta)+1\right) d \zeta\right|+\left|\log \left(f\left(\psi\left(z_{0}\right)\right)\right)+z_{0}\right| \\
& \leq \sup _{w \in\left[z_{0}, z\right]}\left\{\left|\frac{f^{\prime}(\psi(w))}{f(\psi(w))} \cdot \psi^{\prime}(w)+1\right|\right\} \cdot\left|z-z_{0}\right|+C^{\prime} \\
& \leq \varepsilon \cdot\left|z-z_{0}\right|+C^{\prime} \\
& \leq \varepsilon \cdot|z|+C
\end{aligned}
$$


where $C^{\prime}=\left|\log \left(f\left(\psi\left(z_{0}\right)\right)\right)+z_{0}\right|$ and $C>0$ depend only on $z_{0} ;\left[z_{0}, z\right]$ denotes the straight line segment in $F$ connecting $z_{0}$ to $z$. It follows that $\log (f(\psi(z))) \in B_{\varepsilon|z|+C}(-z)$ and

$$
\operatorname{Re}(\log (f(\psi(z))))<-\operatorname{Re}(z)+\varepsilon|z|+C .
$$

Since $\operatorname{Im}(z)$ does not depend on $t$, we have that $|z| / \operatorname{Re}(z) \rightarrow 1$ as $t \rightarrow \infty$ and the right hand side of inequality (5) converges to $-\infty$. Hence, exponentiating (5) yields $\lim _{t \rightarrow+\infty} f(\psi(z))=0$.

Analogous estimates hold for sufficiently large imaginary parts if $V$ is parabolic of type II. If $V$ is hyperbolic, sufficiently large $h$ will permit a construction as above. This finishes the proof.

REMARK. In fact, we not only show the existence of an asymptotic path to 0 for $f$ in $V$, but even that $V$ has an $N_{f}$-invariant open subset in which $f$ converges to 0 along $N_{f}$-orbits. This is another similarity between immediate basins and their virtual counterparts.

\section{AcKnowledgements}

We thank Adrien Douady for raising the question of a relation between virtual immediate basins and asymptotic values and Dierk Schleicher for his helpful comments and his support. We also thank Walter Bergweiler and Alexandre Eremenko for several interesting discussions in which we learned a lot about transcendental functions.

\section{REFERENCES}

[BKL] N. Baker, J. Kotus and Y. Lü, 'Iterates of meromorphic functions IV: critically finite functions', Results in Mathematics (3/4) 22 (1992) 651-656.

[BF] K. Barański and N. Fagella, 'Univalent Baker domains', Nonlinearity (3) 14 (2001) 411-429.

[Be1] W. Bergweiler, 'Iteration of meromorphic functions', Bulletin of the AMS 29 (1993) 151-188.

[Be2] W. Bergweiler, 'On the Julia set of analytic self-maps of the punctured plane', Analysis 15 (1995) 251-256.

[BDL] W. Bergweiler, D. Drasin, J. Langley, 'Baker domains for Newton's method', preprint, ArXiv:math.CV/0601496

[BE] W. Bergweiler and A. Eremenko, 'On the singularities of the inverse to a meromorphic function of finite order', Rev. Mat. Iberoamericana 11 (1995) 355-373.

[BHK] W. Bergweiler, F. von Haeseler, H. Kriete, H.-G. Meier and N. Terglane, 'Newton's method for meromorphic functions', in: Complex Analysis and its Applications, Pitman Res. Notes Math. Ser. 305 (1994) 147-158.

[Co] C. Cowen, 'Iteration and the solution of functional equations for functions analytic in the unit disk', Transactions of the AMS 256 (1981) 69-95.

[E1] A. Epstein, 'Algebraic dynamics I: contraction and finiteness principles', preprint.

[E2] A. Epstein, 'Algebraic dynamics II: finiteness and transversality principles', manuscript in preparation.

[E3] A. Epstein, 'Algebraic dynamics III: transversality and rigidity principles', manuscript in preparation.

[Ma] S. Mayer, 'Newton's method for transcendental functions', Diplomarbeit TU München (2002).

[MS] S. Mayer and D. Schleicher, 'Immediate and virtual basins of Newton's method for entire functions', Annales de l'institut Fourier, to appear. ArXiv:math.DS/0403336 
VIRTUAL IMMEDIATE BASINS OF NEWTON MAPS AND ASYMPTOTIC VALUES 15

[Mi] J. Milnor, Dynamics in one complex variable Vieweg, Braunschweig/Wiesbaden, (2000).

[Ko] H. König, 'Conformal conjugacies in Baker domains', Journal of the London Mathematical Society (2) 59 (1999) 153-170.

[RS] P. Rippon and G. Stallard, 'Iteration of a class of hyperbolic meromorphic functions', Proceedings of the AMS (11) 127 (1999) 3251-3258.

[RuS] J. Rückert and D. Schleicher, 'Virtual and immediate basins for Newton maps of entire functions', preprint, ArXiv:math.DS/0505652

[Sh] M. Shishikura, 'The connectivity of the Julia set and fixed points', preprint, IHES, 1990 .

Université Paul Sabatier, 118 route de Narbonne, 31062 Toulouse, France

E-mail address: buff@picard.ups-tlse.fr

International University Bremen, Campus Ring 12, 28759 Bremen, Germany

E-mail address: j.rueckert@iu-bremen.de 